\title{
Contribution of Informed Reference Prior Using Primary and Summary Measure Data in Bayesian Meta-Analysis
}

\author{
Most. Sifat Muntaha Soni and Md. Belal Hossain* \\ Department of Statistics, Biostatistics and Informatics, Dhaka University, Dhaka - 1000, Bangladesh.
}

(Received: 14 November 2012; Accepted: 28 October 2014)

\begin{abstract}
When meta-analysis includes a small number of trials, inferences are sensitive to the choice of prior distributions for between-study heterogeneity. The common practice is to use vague prior but inferences depend on the degree of vagueness. Pullenayegum (2011) proposed an informed reference prior for between-study heterogeneity of binary outcomes. We employ a model for applying this prior for both primary outcome and summary measure data in Bayesian meta-analysis. We have found the same inference using both primary outcome and summary measure data. This study also suggests that the informed reference prior for between-study heterogeneity represents more relevant conclusion as compared to commonly used prior distributions.
\end{abstract}

Keywords: Informed reference prior; between-study heterogeneity; primary outcome; summary measure; Bayesian metaanalysis.

\section{Introduction}

Meta-analysis is the collection and subsequent analysis of results from numerous studies. Bayesian meta-analysis contains uncertainty for all parameters. So, random effects model automatically incorporated. When meta-analysis contains large number of studies or trials, the choice of prior distribution for between-study variance is easy. The vague or non-informative prior is commonly used, because we allow the data to dominate. In case of small number of trials, inference depends on the vagueness. Browne and Draper ${ }^{1}$ investigated taking a gamma distribution on the precision and uniform distribution on the variance. But it leads to substantial bias and poor coverage in case of small number of studies.

The popularity of Bayesian approach is being increasing in medical statistics. Bayesian models are used in many metaanalysis settings those previously adopted in frequentist formulation. There are some advantages of using Bayesian approach such as full allowance for all parameters uncertainty in the model, the ability to include other relevant information, the ability to extend the models to accommodate more complex situations. Standard methodology of meta-analysis features a choice between a fixed effects model or random effects model. The random effects model gives an estimate of the between study variance. This estimate is obtained by DerSimonian and Laird ${ }^{2}$ inverse variance approach. In this approach weights are the reciprocal of the sum of the between and within study variances. Uncertainty about between study variance is not accounted here. It is important to consider the uncertainty in particular case. For example, when the numbers of studies are small then variances are likely to be estimated subject to considerable uncertainty. Bayesian meta analyses automatically account for the uncertainty about between study variance.

Lambert et $a l^{3}$ assessed the performance of 13 different prior distributions for between study variance. They have found that the point estimates of the pooled log-odds ratio are similar for these 13 different prior distributions, but differ in the width of the $95 \%$ credible intervals. Pullenayegum ${ }^{4}$ proposed an informed reference prior for between-study variance in meta-analysis of binary outcome. In this paper, we show how this prior distribution can be used for both primary and summary measure data in practice as well as the contribution of this prior on inference.

Next, we describe the informed reference prior, the model for using the informed reference prior. We show the model for both primary outcome data and summary measure data. We also discuss a real its example by applying this model. Finally, we cover discussion and conclusion within the context of Bayesian meta-analysis methodology.

\section{Informed Reference Prior for Between-Study Variance}

The Bayesian meta-analysis requires various kinds of uncertainty in the model. These incorporated via the construction of prior distribution. In order to maintain some element of objectivity, vague prior distribution is usually used for overall mean. It is well known that within-study variance is assumed to be known and take the values of the observed within-study variance. Inferences may be sensitive to the choice of prior distribution for between-study variance in case of small number of studies.

Pullenayegum $^{4}$ proposed an informed reference prior for between-study variance. For deriving this prior, he considered lognormal model for between study variance. Initially this model was fitted without covariates and then expanded to include review level covariates for $\theta$. In informed reference prior, the regression coefficient for the logarithmic term and the intercept is used for reducing collinearity between the offsets. When logarithmic transformation of $\theta$ in the covariates for $\log \left(\tau^{2}\right)$ is used, an offset is needed to avoid singularities at $\theta=0$. The offset is treated as stochastic with a $\operatorname{Gamma}(0.1,0.1)$ and its posterior mean was obtained as 0.001 . This value is used as the offset value. Pullenayegum ${ }^{4}$ included 314 studies and used simulation study for finding posterior means for the parameters. His study showed that the values of regression coefficients for assessing the impact of review level covariates are -2.48 and 1.34 and the standard deviation of the lognormal distribution is 0.74 . 
Hence, the informed reference prior for between-study variance $\left(\tau^{2}\right)$ in conjunction with vague prior for overall mean $(\theta)$ is

$$
\begin{gathered}
\log \left(\tau^{2}\right) \sim N\left(-2.48+1.34 * \log ((0.0001+|\theta|) /(0.0001+0.5)), \sigma_{\tau}=0.74\right) \\
\theta \sim N(0,100) .
\end{gathered}
$$

\section{Methods}

The methods described in this section are presented in the context of meta-analysis of clinical trials. Our main purpose is to show how the informed reference prior can be used in practice with its contribution. Bayesian meta-analysis can be done using primary outcomes as well as summary measures. Here, we use both of them. We have presented different models for applying these. There are two possible formulations exist for modeling binary data. The first one is that the observed responses are modeled using binomial distributions with conjugate beta distribution. Another formulation is that the observed responses in each trial are assumed to follow a binomial distribution and then a suitable transformation is used frequently logit in nature to the risk parameters. We use the method for log-odds scale.

Consider a randomized controlled trial, $r_{i}^{c}$ and $r_{i}^{t}$ be the observed number of responses out of $n_{i}^{c}$ and $n_{i}^{t}$ individuals in treatment and control group respectively. The first level of the Bayesian random effects model using primary outcomes is

$$
\begin{array}{r}
r_{i}^{c} \sim \operatorname{Bin}\left(n_{i}^{c}, \pi_{i}^{c}\right) \\
r_{i}^{t} \sim \operatorname{Bin}\left(n_{i}^{t}, \pi_{i}^{t}\right),
\end{array}
$$

where $\pi_{i}^{c}$ and $\pi_{i}^{t}$ be the two unknown risk parameters in the two groups. Using logit transformation of risk parameter we have

$$
\log i t\left(\pi_{i}^{t}\right)=\mu_{i}+\theta_{i},
$$

where $\theta_{i}=\log i t\left(\pi_{i}^{t}\right)-\log i t\left(\pi_{i}^{c}\right)$ is the log-odds ratio which is our parameter of interest and it is assumed to be approximately normally distributed. Therefore

$$
\theta_{i} \sim N\left(\theta, \tau^{2}\right),
$$

where $\theta$ is the overall mean effect on a log ratio scale and $\tau^{2}$ is a measure of the between-study heterogeneity. The informed reference prior for between study variance is

$$
\begin{aligned}
\log \left(\tau^{2}\right) \sim N(-2.48+1.34 & \left.* \log ((0.0001+|\theta|) /(0.0001+0.5)), \sigma_{\tau}=0.74\right) \\
\theta & \sim N(0,100) .
\end{aligned}
$$

A problem of zero cells arises in case of primary outcome data. To resolve this problem, a correction is used ${ }^{5}$. The advantage of using summary measure is that we do not need any correction.

Let $\mathrm{d}_{\mathrm{i}}$ be the point estimate of true treatment effect $\theta_{i}$ in trials i. In the random effects approach, the $\theta_{i}$ 's are assumed to be drawn from a common normal distribution. Thus the model is

$$
\begin{aligned}
& d_{i} \sim N\left(\theta_{i}, \operatorname{SE}\left(d_{i}\right)^{2}\right) \\
& \theta_{i} \sim N\left(\theta, \tau^{2}\right)
\end{aligned}
$$

$\log \left(\tau^{2}\right) \sim N\left(-2.48+1.34 * \log ((0.0001+|\theta|) /(0.0001+0.5)), \sigma_{\tau}=0.74\right)$

$$
\theta \sim N(0,100) \text {. }
$$

WinBUGS version 1.4 is used to analyse these models.

\section{Applications}

The informed reference prior is proposed for small number of trials. In our study, we use four large trials. Cannon et $a l^{6}$ conducted a meta-analysis that shows the impact of highdose versus standard-dose of statins in preventing death or myocardial infarction (MI). We have used primary outcomes from Cannon el al ${ }^{6}$.

\section{1) Frequentist analysis}

The log odds ratios and their respective standard errors, confidence intervals for the four randomized control trials are reported in Table 1. These values are plotted in Fig 1. Forest plot in Figure 1 is commonly used method for reporting the presence of heterogeneity. This plot highlights the difference in precision between estimates from different studies. Each study effect estimate i.e., $\log \mathrm{OR}$ and respective confidence interval are plotted on one set of axis. The vertical line on the plot corresponds to a logOR of zero where treatment and control group are equally effective. The pooled estimate together with its confidence interval is also plotted below the individual study results in the figure. The size of the plotting symbol used to mark the point estimate from each study is made proportional to the reciprocal of the variance of the estimate. The more influential trials in a meta-analysis show the largest plotting symbols. The analysis is done by using meta module of STATA version 11 software.

From the forest plot, we have observed that the majority of confidence intervals do include zero and hence individually inconclusive. Statistically significant beneficial effect of treatment is observed in TNT study. A to Z are the least precise study i.e., least influential as a results of their widest confidence intervals. The variability between estimates on the plot highlights the homogeneity between the studies. A meta-analysis of small number of trials represents homogeneity in most of the time. But, a meta-analysis with large number of trials may represent heterogeneity. The combined estimate is far from zero, indicating that the treatment i.e., high dose statin has an impact on decreasing death or myocardial infarction. The $95 \%$ confidence interval around this estimate is reasonably narrow, indicating precise pooled result. This interval reflects the fact that only a small number of studies are pooled. The conclusion that the treatment has an impact on decreasing death or myocardial infarction appears reasonable.

From the result of Table 2, we have observed that the pooled log-odds ratio is -0.18 and the test statistic $Q=1.141^{7}$. The test statistic of 1.141 is compared to a chi square distribution on 3 degrees of freedom which gives a p-value which is statistically insignificant (p-value=0.767). A test for the existence of heterogeneity depends on the number of studies in the meta-analysis. The test has poor power in the common situation of few studies. Our study includes only four trials. So, the test of heterogeneity does not provide a relevant summary of the extent to the impact on the metaanalysis. If the number of trials increases then the Q-values also increases as always. 


\section{2) Bayesian meta-analysis using primary outcomes}

It is well known that meta-analysis can be done using primary outcomes as well as summary measures. We apply informed reference prior in both case. For using primary outcomes, model 1 is used.

Bayesian analysis has been implemented by WinBUGS version 1.4 software $^{8,9,10}$. Before making inference, we check convergence of the sample iterations. The trace plot, autocorrelation, and MC error are used for checking convergence. We report median because of its stability.

We have found the pooled odds-ratio is 0.85 ( $\mathrm{CrI} 0.70$ to 0.94) after converting to odds ratio scale. That means the risk of death or $\mathrm{MI}$ is $15 \%$ lower for patients assign to the high dose than the patients assigned to standard dose. We also compute the probability of high-dose statin benefitted is 0.9979 .

Now we use vague prior instead of informed reference prior for between-study variance. The commonly used vague prior for between-study variance is $\operatorname{Uniform}(0,2)$. Applying Uniform $(0,2)$ as a vague prior, we have found the pooled odds ratio is 0.83 (CrI 0.70 to 0.99 ) (Table 4). This indicates that the risk of death or MI is $17 \%$ lower for patients assign to the high dose than the patients assigned to standard dose. The probability of high-dose statin benefited is 0.977 .

\section{3) Bayesian meta-analysis using summary measures}

Model 2 is used for summary measures. As before, firstly we use informed reference prior for between-study variance and secondly we use vague prior.

We have found that the pooled log-odds ratio is -0.164 (Table 5) and standard deviation of pooled log-odds ratio is 0.07551 . Thus the odds ratio is 0.85 , which is same as we get from the primary outcome. We have found the probability of high-dose statin effective is 0.9984

We also present the results using vague prior in Table 6. The pooled log-odds ratio using summary measures is -0.1805 . Converting to odds scale, we have odds ratio is 0.83 as like as model 1 . The probability of high-dose statin effective is 0.9771 .

Table 1. Impact of high-dose statin vs Standard dose, Source: (Cannon et al. 2006)

\begin{tabular}{|c|c|c|c|c|c|c|c|c|}
\hline \multirow[t]{2}{*}{ Study Name } & \multicolumn{2}{|c|}{ High-dose } & \multicolumn{2}{|c|}{ Standard-dose } & \multirow[t]{2}{*}{$\log (\mathrm{OR})$} & \multirow[t]{2}{*}{$\mathrm{SE}(\log (\mathrm{OR}))$} & \multicolumn{2}{|c|}{$95 \% \mathrm{CI}$} \\
\hline & Total & Death & Total & Death & & & Lower & Upper \\
\hline PROVE IT & 2099 & 147 & 2063 & 172 & -0.189 & 0.1169 & -0.42 & 0.04 \\
\hline A to $\mathrm{Z}$ & 2265 & 205 & 2232 & 235 & -0.168 & 0.1006 & -0.36 & 0.03 \\
\hline TNT & 4995 & 334 & 5006 & 418 & -0.240 & 0.0763 & -0.39 & -0.09 \\
\hline IDEAL & 4439 & 411 & 4449 & 463 & -0.130 & 0.0714 & -0.27 & 0.01 \\
\hline
\end{tabular}

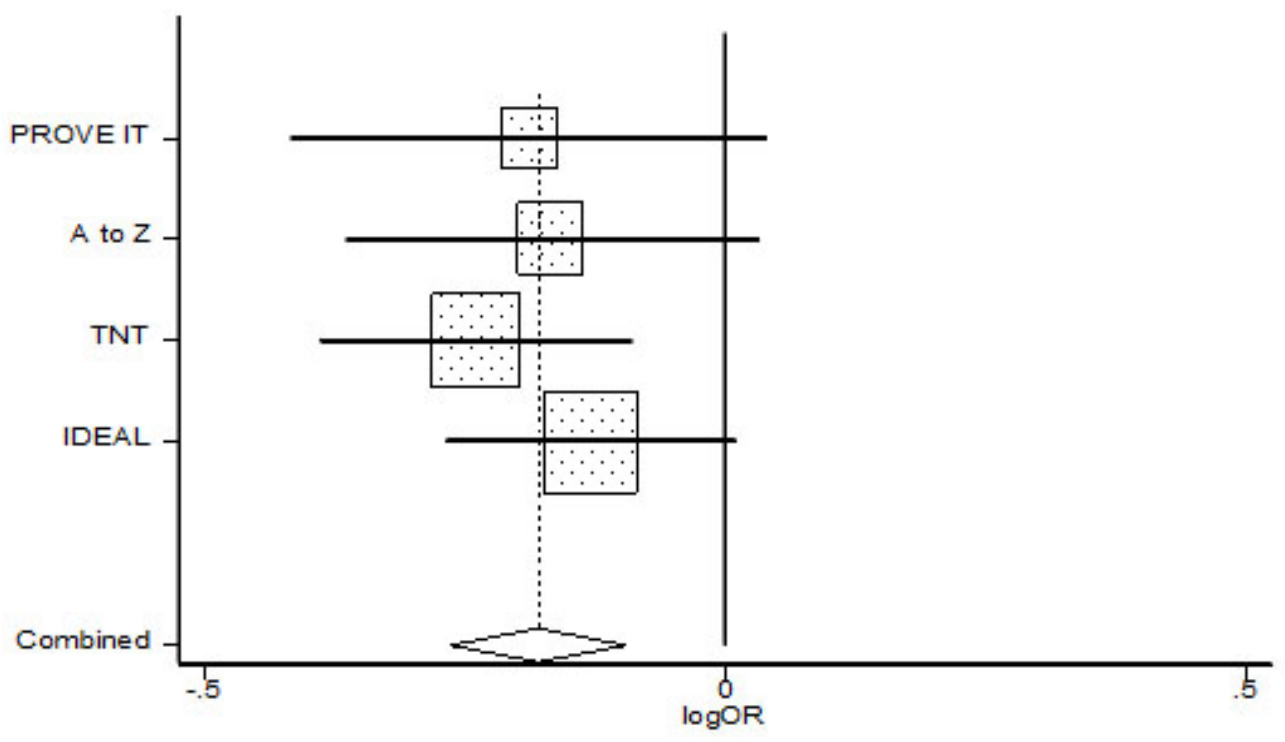

Fig. 1. Forest plot for the impact of Statin. 
Table 2. Results of frequentist analysis for small data set

\begin{tabular}{|c|c|c|c|c|c|c|}
\hline \multicolumn{7}{|c|}{ Meta Analysis } \\
\hline \multirow[t]{2}{*}{ Method } & \multirow{2}{*}{$\begin{array}{c}\text { Pooled } \\
\text { Est }\end{array}$} & \multicolumn{2}{|c|}{$95 \% \mathrm{CI}$} & \multicolumn{2}{|c|}{ Asymptotic } & \multirow{2}{*}{$\begin{array}{l}\text { Number of } \\
\text { studies }\end{array}$} \\
\hline & & Lower & Upper & Z-value & p-value & \\
\hline Fixed effects model & -0.180 & -0.264 & -4.178 & -4.178 & 0.000 & \multirow[t]{2}{*}{4} \\
\hline Random effects model & -0.180 & -0.264 & -4.178 & -4.178 & 0.000 & \\
\hline \multicolumn{7}{|c|}{$\begin{array}{l}\text { Test for heterogeneity: } Q=1.141 \text { on } 3 \text { degrees of freedom }(p=0.767) \\
\text { Moment based estimate of between studies variance }=0.000\end{array}$} \\
\hline \multirow[t]{2}{*}{ Study } & \multicolumn{2}{|c|}{ Weights } & Study & \multicolumn{2}{|c|}{$95 \% \mathrm{CI}$} & \\
\hline & Fixed & Random & Est & Lower & Upper & \\
\hline Prove-it & 73.22 & 73.22 & -0.19 & -0.42 & 0.04 & \\
\hline A to $\mathrm{Z}$ & 98.82 & 98.82 & -0.17 & -0.36 & 0.03 & \\
\hline TNT & 171.85 & 171.85 & -0.24 & -0.39 & -0.09 & \\
\hline Ideal & 196.38 & 196.38 & -0.13 & -0.27 & 0.01 & \\
\hline
\end{tabular}

Table 3. Posterior summaries using primary outcomes and informed reference prior for between-study variance

\begin{tabular}{|llllll|}
\hline Parameters & MC error & Median & $\begin{array}{l}\text { Standard } \\
\text { deviation }\end{array}$ & 95\%CrI & P( $\theta<0)$ \\
\hline Pooled log-odd ratio( $\theta)$ & 0.001314 & -0.1652 & 0.07726 & $(-0.3579,-0.0589)$ & 0.9979 \\
log-odds ratio[1] & 0.001287 & -0.1733 & 0.08925 & $(-0.3645,-0.01399)$ \\
log-odds ratio [2] & 0.001253 & -0.1642 & 0.07966 & $(-0.329,-0.01968)$ \\
log-odds ratio [3] & 0.001145 & -0.2095 & 0.07002 & $(-0.3494,-0.07937)$ \\
log-odds ratio [4] & $8.65 E-04$ & -0.1382 & 0.06121 & $(-0.2594,-0.02063)$ \\
$\tau^{2}$ & $3.32 \mathrm{E}-04$ & 0.01133 & 0.02078 & $(0.002532,0.06255)$ \\
\hline
\end{tabular}

Table 4. Posterior summaries using primary outcomes and Uniform(0, 2) for between-study variance

\begin{tabular}{|l|l|l|l|l|l|}
\hline Parameters & MC error & Median & Standard Deviation & $95 \%$ CrI & $\mathrm{P}(\theta<0)$ \\
\hline Pooled log-odds ratio( $\theta)$ & $7.94 \mathrm{E}-04$ & -0.1833 & 0.09747 & $(-0.3507,-0.0105)$ & 0.977 \\
\hline log-odds ratio[1] & $6.13 \mathrm{E}-04$ & -0.1855 & 0.0761 & $(-0.3451,-0.03241)$ & $(-0.3207,-0.03458)$ \\
\hline log-odds ratio[2] & $6.00 \mathrm{E}-04$ & -0.1795 & 0.07049 & $(-0.3412,-0.09379)$ & \\
\hline log-odds ratio[3] & $5.58 \mathrm{E}-04$ & -0.2057 & 0.06254 & $(-0.2723,-0.0344)$ & \\
\hline log-odds ratio[4] & $5.81 \mathrm{E}-04$ & -0.1627 & 0.06008 & $(6.75 \mathrm{E}-06,0.2198)$ & \\
\hline$\tau^{2}$ & 0.001349 & 0.004038 & 0.1497 & &
\end{tabular}

Table 5. Posterior summaries using summary measures and informed reference prior for between-study variance

\begin{tabular}{|llllll|}
\hline Parameters & MC error & Median & $\begin{array}{l}\text { Standard } \\
\text { deviation }\end{array}$ & 95\% CrI & $P(\theta<0)$ \\
\hline Pooled log-odds ratio $(\theta)$ & 0.001136 & -0.164 & 0.07551 & $(-0.3352,-0.05498)$ & 0.9984 \\
$\tau^{2}$ & $4.18 \mathrm{E}-04$ & 0.00982 & 0.02383 & $(0.00175,0.06239)$ & \\
\hline
\end{tabular}


Table 6. Posterior summaries using summary measures and Uniform( $(0,2)$ for between-study variance

\begin{tabular}{|llllll|}
\hline Parameters & MC error & Median & $\begin{array}{l}\text { Standard } \\
\text { deviation }\end{array}$ & $95 \%$ CrI & $P(\theta<0)$ \\
Pooled log-odds ratio $(\theta)$ & 0.002139 & -0.1805 & 0.1094 & $(-0.3658,-0.00806)$ & 0.9771 \\
$\tau^{2}$ & 0.003194 & 0.00419 & 0.1774 & $(8.55 E-06,0.2024)$ & \\
\hline
\end{tabular}

\section{Discussion and Conclusion}

Clinical trials are good candidates for meta-analysis. This paper has presented Bayesian meta-analysis of clinical trials with binary outcomes using odds ratio scale. The method presented allows the investigation of source of heterogeneity. The specification of prior distribution for between-study variance is one of the most controversial aspects of Bayesian meta-analysis. It is very important in case of small number of studies. In this study, we use informed reference prior for between-study variance. We have also discussed a model for using this prior distribution and showed how to implement it using WinBUGS.

The inferences applying informed reference prior for primary outcomes and summary measures are the same. For making a comparison with informed reference prior, we use vague prior for between-study variance. We have observed the pooled odds-ratio is 0.85 ( $\mathrm{CrI} 0.70$ to 0.94 ) using informed reference prior, but in case of vague prior the pooled odds-ratio is 0.83 (CrI 0.70 to 0.99 ). That means, the high-dose statin has $15 \%$ less risk of death or myocardial infarction than standard-dose when applying informed reference prior for between-study variance. Applying vague prior the high-dose statin has $17 \%$ less risk of death or myocardial infarction as compared to standard-dose. As the outcome is serious in most cases i.e., death or myocardial infarction, more careful attention is required for taking decision. The length of credible interval is smaller for informed reference prior as compared to vague prior. Therefore, informed reference prior gives more reliable inference than vague prior. The informed reference prior may be the solution to overcome the limitations of vague prior. In case of small number of trials with binary outcome informed reference prior improves the inference in Bayesian meta-analysis.

\section{References}

1. Browne, W. J. and D. Draper, 2004. A comparison of Bayesian and likelihood method for fitting multi level models. Nothigham Statistics Report, 01-04.

2. DerSimonian, R. and N. Laird, 1986. Meta-analysis in clinical trials. Control Clin Trials, 7, 177-88.

3. Lambert, P. C., A. J. Sutton, P. R. Burton, K. R. Abrams and D. R. Jones, 2005. How vague is vague? A simulation study of the impact of the use of vague prior distributions in MCMC using WinBUGS. Statistics in Medicine, 24, 2401-2428.

4. Pullenayegum, E. M., 2011. An informed reference prior for between-study heterogeneity in meta-analyses of binary outcomes. Statistics in Medicine, 30, 3082-3094.

5. Gart, J. J. and J. R. Zweifel, 1967. On the bias of various estimators of the logit and its variance, with application to quantal bioassay. Biometrica, 54, 471-5.

6. Cannon, C. P., B. A. Steinberg, S. A. Murphy, J. L. Mega and E. Braunwald, 2006. Meta-analysis of cardiovascular outcome trials comparing intensive versus moderate statin therapy. Journal of the American College of Cardiology, 48(3), 438445.

7. Cochran, W. G., 1954. The combination of estimates from different experiments. Biometrics, 10, 101-29.

8. Lab 8: Introduction to WinBUGS. www.biostat.jhsph.edu.

9. Spiegellhalter, D., A. Thomas, N. Best and D. Lunn, 2003. WinBUGS User Manual version 1.4.

10. Lunn, D. J., A. Thomas, N. Best and D. Spiegelhalter, 2000. WinBUGS-a Bayesian modeling framework: concepts, structure, and extensibility. Statistics and Computing, 10, 325-337. 
